and making a set of five assumptions. It appears that the most general solution, when $n$ is greater than 2 , is $\varphi^{-1} f^{r} \varphi(x)$, where the integer $r$ is prime to $n$. The case $n=2$ is discussed separately and a simple algorism is given for reducing all differentiable functions of order 2 to a single type.

F. N. Cole, Secretary.

\title{
THE LEGENDRE CONDITION FOR A MINIMUM OF A DOUBLE INTEGRAL WITH AN ISOPERI- METRIC CONDITION.
}

BY DR. CHARLES ALBERT FISCHER.

(Read before the American Mathematical Society, February 28, 1914.)

THE Legendre, or second necessary, condition for a minimum of a double integral, where there is no isoperimetric condition, has been derived by Kobb, ${ }^{*}$ where the equations of the surfaces involved are in parametric form, and by Mason, $\dagger$ where $x$ and $y$ are the independent variables. The analogous condition for the isoperimetric problem has been proved to be sufficient to insure a permanent sign to the second variation, $\ddagger$ but it has not been proved to be necessary.

In the present paper this condition,

$h_{p p}(x, y, z, p, q ; \lambda) h_{q q}(x, y, z, p, q ; \lambda)-h_{p q}{ }^{2}(x, y, z, p, q ; \lambda) \geqq 0$, or expressed in parametric form,

$$
\begin{aligned}
H_{11}\left(x, y, z, x_{u}, x_{v}, \cdots, z_{v} ; \lambda\right) H_{22}\left(x, y, z, x_{u}, x_{v}, \cdots, z_{v} ; \lambda\right) & \\
- & H_{12}{ }^{2}\left(x, y, z, x_{u}, x_{v}, \cdots, z_{v} ; \lambda\right) \geqq 0,
\end{aligned}
$$

is proved to be necessary for either a maximum or a minimum.

Given two functions $f(x, y, z, p, q)$ and $g(x, y, z, p, q)$ and a surface

$$
S: \quad z=z(x, y)
$$

* "Sur les maxima et les minima des intégrales doubles," Acta Mathematica, vol. 16 (1892), p. 108 .

$\dagger$ "A necessary condition for an extremum of a double integral," BuLLETIN, vol. 13 (1907), p. 293.

$\ddagger$ Kobb, Acta Mathematica, vol. 17 (1893), p. 331 . 
which satisfies the Lagrange differential equation

$$
\begin{aligned}
h_{z}(x, y, z, p, q ; \lambda)-\frac{\partial}{\partial x} h_{p}(x, y, z, p, q ; \lambda) & \\
& -\frac{\partial}{\partial y} h_{q}(x, y, z, p, q ; \lambda)=0 \text {; }^{*}
\end{aligned}
$$

it is desired to find a second condition which must be satisfied if the surface $S$ gives a value to the integral

$$
J=\iint_{\Omega} f(x, y, z, p, q) d x d y
$$

as small as that given by any other admissible surface in the neighborhood of $S$. The region $\Omega$ is assumed to be bounded by a curve $L$ of class $D^{\prime}, \dagger$ without double points, and the number of its intersections with any line parallel to either of the axes is assumed to be less than a fixed constant. The function $z(x, y)$ is assumed to be of class $C^{\prime \prime}$ in $\Omega$, as are also the functions $f$ and $g$ in the neighborhood of $S$. A surface is said to be admissible if it is of class $D^{\prime}$, intersects $S$ along a space curve which projects into $L$, and gives the same value as $S$ to the double integral

$$
K=\iint_{\Omega} g(x, y, z, p, q) d x d y .
$$

It will also be assumed that $z(x, y)$ is not a solution of the equation

(3) $g_{z}(x, y, z, p, q)-\frac{\partial}{\partial x} g_{p}(x, y, z, p, q)$

$$
-\frac{\partial}{\partial y} g_{q}(x, y, z, p, q)=0 .
$$

If a one parameter family of admissible surfaces

$$
\bar{S}:
$$

$$
z=z(x, y)+\epsilon \bar{\zeta}(x, y, \epsilon)
$$

is given, where the function $\bar{\zeta}(x, y, 0)$ and its partial derivative $\zeta_{\epsilon}(x, y, 0)$ are of class $D^{\prime}$, and this value substituted for $z$ in the function $f$, the first variation of $J$ must vanish because of

* Bolza: Vorlesungen über Variationsrechnung, p. 662.

† Bolza, loc. cit., p. 63. 
equation (1). The second variation is found to be

$$
\begin{aligned}
\delta^{2} J= & \epsilon^{2} \iint_{\Omega}\left(f_{z z} \bar{\zeta}^{2}+2 f_{z p} \overline{\zeta \zeta}_{x}+2 f_{z q} \overline{\zeta \zeta}_{y}+f_{p p} \bar{\zeta}_{x}^{2}\right. \\
& \left.+2 f_{p q} \bar{\zeta}_{x} \bar{\zeta}_{y}+f_{q q} \bar{\zeta}_{y}^{2}+f_{z} \bar{\zeta}_{\epsilon}+f_{p} \bar{\zeta}_{x \epsilon}+f_{q} \bar{\zeta}_{y \epsilon}\right) d x d y
\end{aligned}
$$

Since the surfaces considered are admissible, $\delta^{2} K$, which can be evaluated in the same way, vanishes. If it is multipled by $\lambda$ and added to $\delta^{2} J$, and then Green's theorem* is applied, equation (4) becomes

$$
\begin{aligned}
\delta^{2} J=\epsilon^{2} \iint_{\Omega}\left(h_{z z} \bar{\zeta}^{2}+\right. & \left.2 h_{z p} \bar{\zeta} \bar{\zeta}_{x}+\cdots+h_{q q} \bar{\zeta}_{y}^{2}\right) d x d y \\
& +\epsilon^{2} \iint_{\Omega}\left(h_{z}-\frac{\partial}{\partial x} h_{p}-\frac{\partial}{\partial y} h_{q}\right) \bar{\zeta}_{\epsilon} d x d y
\end{aligned}
$$

where as usual $h=f+\lambda g$. The last integral vanishes on account of equation (1), leaving

$$
\delta^{2} J=\epsilon^{2} \iint_{\Omega}\left(h_{z z} \bar{\zeta}^{2}+2 h_{z p} \overline{\zeta \zeta}_{x}+\cdots+h_{q q} \bar{\zeta}_{y}^{2}\right) d x d y .
$$

It will now be proved that if there is a point on $S$ where the inequality

$$
h_{p p} h_{q q}-h_{p q}^{2}<0
$$

is satisfied, the function $\bar{\zeta}(x, y, \epsilon)$ can be chosen in such a way that $\delta^{2} J$ will be negative, and consequently there is no minimum.

If there is such a point there must be a region including the point where inequality (6) is satisfied. Two distinct points $P_{0}$ and $P_{0}^{\prime}$ in such a region can then be chosen, whose coordinates will be called $x_{0}, y_{0}, z\left(x_{0}, y_{0}\right)$ and $x_{0}{ }^{\prime}, y_{0}{ }^{\prime}, z\left(x_{0}{ }^{\prime}, y_{0}{ }^{\prime}\right)$. Since $z(x, y)$ is not a solution of equation (3), it can be assumed that

$$
\begin{aligned}
g_{z}\left(x_{0}{ }^{\prime}, y_{0}{ }^{\prime}, \cdots\right)-\frac{\partial}{\partial x_{0}{ }^{\prime}} g_{p}\left(x_{0}{ }^{\prime}, y_{0}{ }^{\prime}, \cdots\right) & \\
& -\frac{\partial}{\partial y_{0}{ }^{\prime}} g_{q}\left(x_{0}{ }^{\prime}, y_{0}{ }^{\prime}, \cdots\right) \neq 0 .
\end{aligned}
$$

\footnotetext{
* Bolza, loc. cit., p. 654 .
} 
The expression

$h_{p p}\left(x_{0}, y_{0}, \cdots\right) \cos ^{2} \alpha+2 h_{p q}\left(x_{0}, y_{0}, \cdots\right) \cos \alpha \sin \alpha$

$$
+h_{q q}\left(x_{0}, y_{0}, \cdots\right) \sin ^{2} \alpha
$$

must vanish for two positive values of $\alpha$ less than $\pi$, and changes its sign when and only when $\alpha$ passes through one of them. It follows that a positive constant $k^{2}$ and a finite interval can be chosen in such a way that if $\alpha_{1}$ and $\alpha_{2}$ are any two angles in the interval, the inequalities

(8) $h_{p p} \cos ^{2} \alpha_{i}+2 h_{p q} \cos \alpha_{i} \sin \alpha_{i}+h_{q q} \sin ^{2} \alpha_{i}<-k^{2}$

$$
(i=1,2) \text {, }
$$

are satisfied at $P_{0}$, and since $h_{p p}, h_{p q}$ and $h_{q q}$ are continuous, they are satisfied at every point of $S$ in a neighborhood of $P_{0}$. If there is given any positive constant $\delta$ it is possible to select two distinct angles $\alpha_{1}{ }^{\prime}$ and $\alpha_{2}{ }^{\prime}$ near to a root of the equation $h_{p p}\left(x_{0}{ }^{\prime}, y_{0}{ }^{\prime}, \cdots\right) \cos ^{2} \alpha+2 h_{p q}\left(x_{0}{ }^{\prime}, y_{0}{ }^{\prime}, \cdots\right) \cos \alpha \sin \alpha$

$$
+h_{q q}\left(x_{0}{ }^{\prime}, y_{0}{ }^{\prime}, \cdots\right) \sin ^{2} \alpha=0,
$$

such that the inequalities

$$
\begin{aligned}
&\left|h_{p p} \cos ^{2} \alpha_{i}{ }^{\prime}+2 h_{p q} \cos \alpha_{i}{ }^{\prime} \sin \alpha_{i}{ }^{\prime}+h_{q q} \sin ^{2} \alpha_{i}{ }^{\prime}\right|<\delta \\
&(i=1,2),
\end{aligned}
$$

are satisfied at every point of $S$ in a neighborhood of $P_{0}{ }^{\prime}$. For convenience these angles will be chosen so that $\alpha_{2}-\alpha_{1}$ $=\alpha_{2}{ }^{\prime}-\alpha_{1}{ }^{\prime}$.

Two rhombuses $R$ and $R^{\prime}$ will be defined as follows:* $R$ is bounded by the lines

$$
d-u_{1}=0, d-u_{2}=0, d+u_{1}=0, d+u_{2}=0,
$$

where

$$
u_{i}=\left(x-x_{0}\right) \cos \alpha_{i}+\left(y-y_{0}\right) \sin \alpha_{i}, \quad(i=1,2),
$$

and $R^{\prime}$ by the lines

$$
d-u_{1}^{\prime}=0, \quad d-u_{2}^{\prime}=0, \quad d+u_{1}^{\prime}=0, \quad d+u_{2}{ }^{\prime}=0,
$$

where

$$
u_{i}{ }^{\prime}=\left(x-x_{0}{ }^{\prime}\right) \cos \alpha_{i}{ }^{\prime}+\left(y-y_{0}{ }^{\prime}\right) \sin \alpha_{i}{ }^{\prime} .
$$

\footnotetext{
* Compare with Mason, loc. cit., p. 295.
} 
The constant $d$ will be taken so small that $R$ and $R^{\prime}$ do not overlap, and are entirely within the projections on the $x, y$ plane of the respective neighborhoods in which the inequalities (8) and (9) are satisfied. A function $\zeta(x, y)$ will be defined as identically zero outside of $R$, and inside of $R$ as equal to the distance of the point $x, y$ from the nearest side of $R$. That is,

$$
\zeta(x, y)=d \mp u_{i}, \quad\left(i^{\prime}=1,2\right),
$$

where the sign and subscript of $u$ are chosen so as to make $\zeta$ as small as possible. The function $\zeta^{\prime}(x, y)$ will be chosen in the analogous way. Since $\alpha_{2}-\alpha_{1}=\alpha_{2}{ }^{\prime}-\alpha_{1}{ }^{\prime}$ the rhombuses $R$ and $R^{\prime}$ are of the same size and shape and the equation

is satisfied.

$$
\iint_{R} \zeta(x, y) d x d y=\iint_{R^{\prime}} \zeta^{\prime}(x, y) d x d y
$$

The function $\bar{\zeta}(x, y, \epsilon)$ will now be defined by the equation

$$
\epsilon \zeta(x, y, \epsilon)=\epsilon \zeta(x, y)+\epsilon^{\prime}(\epsilon) \zeta^{\prime}(x, y),
$$

where $\epsilon^{\prime}(\epsilon)$ is to be determined by the condition that the surfaces $\bar{S}$ be admissible. The first variation of $K$ is found to be equal to

$$
\begin{aligned}
\iint_{R}\left(g_{z}-\frac{\partial}{\partial x} g_{p}-\frac{\partial}{\partial y} g_{q}\right) \zeta d x d y & \\
& +\frac{d \epsilon^{\prime}(0)}{d \epsilon} \iint_{R^{\prime}}\left(g_{z}-\frac{\partial}{\partial x} g_{p}-\frac{\partial}{\partial y} g_{q}\right) \zeta^{\prime} d x d y=0 .
\end{aligned}
$$

If the mean value theorem is applied to these integrals and the equation is solved for $d \epsilon^{\prime}(0) / d \epsilon$, it becomes

$$
\frac{d \epsilon^{\prime}(0)}{d \epsilon}=-\frac{\left(g_{z}-\frac{\partial}{\partial x} g_{p}-\frac{\partial}{\partial y} g_{q}\right)_{x=\xi, y=\eta},}{\left(g_{z}-\frac{\partial}{\partial x} g_{p}-\frac{\partial}{\partial y} g_{q}\right)_{x=\xi^{\prime}, y=\eta^{\prime}}}
$$

where $\xi, \eta$ is a point in $R$ and $\xi^{\prime}, \eta^{\prime}$ a point in $R^{\prime}$. The denominator cannot vanish if $d$ is sufficiently small, because of inequality (7). Consequently a finite constant $m$ can be chosen such that

$$
\left|\frac{d \epsilon^{\prime}(0)}{d \boldsymbol{\epsilon}}\right|<m .
$$


If the second variation of $K$ is equated to zero, the coefficient of $d^{2} \epsilon^{\prime}(0) / d \epsilon$ is also equal to

$$
\iint_{R^{\prime}}\left(g_{z}-\frac{\partial}{\partial x} g_{p}-\frac{\partial}{\partial y} g_{q}\right) \zeta^{\prime} d x d y \neq 0 .
$$

Consequently this second derivative exists. Therefore the functions

$$
\begin{aligned}
& \bar{\zeta}(x, y, 0)=\zeta(x, y)+\frac{d \epsilon^{\prime}(0)}{d \epsilon} \zeta^{\prime}(x, y), \\
& \zeta_{\epsilon}(x, y, 0)=\frac{d^{2} \epsilon^{\prime}(0)}{d \epsilon^{2}} \zeta^{\prime}(x, y)
\end{aligned}
$$

exist and they have the required continuity.

When $\bar{\zeta}(x, y, \epsilon)$ is determined in this way equation (5) may be written

$$
\begin{aligned}
& \delta^{2} J=\epsilon^{2} \iint_{R+R^{\prime}}\left(h_{z z} \bar{\zeta}^{2}+2 h_{z p} \bar{\zeta} \bar{\zeta}_{x}+2 h_{z q} \bar{\zeta} \bar{\zeta}_{y}\right) d x d y \\
&+ \epsilon^{2} \iint_{R}\left(h_{p p} \cos ^{2} \alpha_{i}+2 h_{p q} \cos \alpha_{i} \sin \alpha_{i}+h_{q q} \sin ^{2} \alpha_{i}\right) d x d y \\
&+\epsilon^{2}\left(\frac{d \epsilon^{\prime}(0)}{d \epsilon}\right)^{2} \iint_{R^{\prime}}\left(h_{p p} \cos ^{2} \alpha_{i}{ }^{\prime}+2 h_{p q} \cos \alpha_{i}{ }^{\prime} \sin \alpha_{i}{ }^{\prime}\right. \\
&\left.+h_{q q} \sin ^{2} \alpha_{i}{ }^{\prime}\right) d x d y,
\end{aligned}
$$

where $i$ takes the values 1 and 2 in the appropriate parts of $R$ and $R^{\prime}$. It can now be easily proved that

$$
\delta^{2} J<-\epsilon^{2} A\left(k^{2}-M d(d+4)\left(1+m^{2}\right)-\delta m^{2}\right),
$$

where $M$ is the largest of the maxima of the numerical values of $h_{z z}, h_{z p}$ and $h_{z q}$, and $A$ is the area of $R .^{*}$ Since $d$ and $\delta$ can be taken as small as is desired without affecting $k^{2}$, they can be taken so small that $\delta^{2} J$ will be negative and there is no minimum.

In a similar way it can be proved that there is no maximum if inequality (6) is satisfied, and the following theorem will be proved:

$A$ necessary condition that the surface $S$ furnish either a maximum or a minimum for the double integral (2), relative to

* Compare with Mason, loc. cit., pp. 295-6. 
the admissible surfaces, is that

$$
h_{p p}(x, y, z, p, q ; \lambda) h_{q q}(x, y, z, p, q ; \lambda)
$$

at every point of $S$.

$$
-h_{p q}{ }^{2}(x, y, z, p, q ; \lambda) \geqq 0,
$$

If the surface $S$ is represented by the parametric equations

$$
x=x(u, v), \quad y=y(u, v), \quad z=z(u, v),
$$

the functions $f$ and $g$ must be replaced by $F\left(x, y z, x_{u}, x_{v}, \cdots\right.$ $\left.z_{v}\right)$ and $G\left(x, y, z, x_{u}, x_{v}, \cdots, z_{v}\right)$ respectively. Then if the equations of $\bar{S}$ are written

$$
\begin{gathered}
x=x(u, v)+\epsilon \xi(u, v, \epsilon), \quad y=y(u, v)+\epsilon \eta(u, v, \epsilon), \\
z=z(u, v)+\epsilon \zeta(u, v, \epsilon),
\end{gathered}
$$

the second variation becomes

$$
\delta^{2} J=\epsilon^{2} \iint_{\Omega}\left(H_{x x} \xi^{2}+2 H_{x y} \xi \eta+\cdots+H_{z_{v} z_{v}} \zeta_{v}{ }^{2}\right) d u d v .
$$

The values of $J$ and $K$ are assumed to be unchanged by any change in the parametric representation of $S$ which leaves the surface $S$ itself invariant. This furnishes a number of relations between the partial derivatives of $F$ and $G$, among which are the following:*

$$
\begin{gathered}
F_{x_{u} x_{u}}=F_{11} X^{2}, \quad F_{x_{u} y_{u}}=F_{12} X Y, \quad F_{x_{u} x_{v}}=F_{12} X^{2}, \\
F_{x_{u} y_{v}}+F_{x_{v} y_{u}}=2 F_{12} X Y, \quad F_{x_{v} x_{v}}=F_{22} X^{2}, \quad F_{x_{v} y_{v}}=F_{22} X Y,
\end{gathered}
$$

and the other formulas derived from these by permuting the letters $x, y, z$ and $X, Y, Z$ in the same way. The functions $F_{11}, F_{12}$ and $F_{22}$ are continuous and $X, Y$ and $Z$ are the direction cosines of the normal to $S$.

It will now be assumed that there is a function $\omega(u, v, \epsilon)$, such that

$$
\begin{aligned}
& \xi(u, v, \epsilon)=\omega(u, v, \epsilon) X \\
& \eta(u, v, \epsilon)=\omega(u, v, \epsilon) Y, \\
& \zeta(u, v, \epsilon)=\omega(u, v, \epsilon) Z .
\end{aligned}
$$

If these values are substituted in the integrand of equation (11), it becomes a quadratic form in $\omega, \omega_{u}, \omega_{v}$. The coefficient

\footnotetext{
* Kneser: Lehrbuch der Variationsrechnung, p. 282.
} 
of $\omega_{u}^{2}$ is seen to be

$$
\begin{aligned}
H_{x_{u} x_{u}} X^{2}+2 H_{x_{u} y_{u}} X Y+H_{y_{u} y_{u}} Y^{2}+2 H_{x_{u} z_{u}} X Z \\
+2 H_{y_{u_{u}} z_{u}} Y Z+H_{z_{u} z_{u}} Z^{2} .
\end{aligned}
$$

Equations (12), with $F$ replaced by $H$, reduce this to the form

$$
H_{11}\left(X^{2}+Y^{2}+Z^{2}\right)^{2}=H_{11} \text {. }
$$

Similarly the coefficients of $\omega_{u} \omega_{v}$ and $\omega_{v}^{2}$ can be proved equal to $2 H_{12}$ and $H_{22}$ respectively. The other coefficients will be called $H_{00}, 2 H_{01}$ and $2 H_{02}$ respectively, and equation (11) becomes

$$
\begin{aligned}
\delta^{2} J=\epsilon^{2} \iint_{\Omega}\left(H_{00} \omega^{2}+2 H_{01} \omega \omega_{u}\right. & +2 H_{02} \omega \omega_{v}+H_{11} \omega_{u}{ }^{2} \\
& \left.+2 H_{12} \omega_{u} \omega_{v}+H_{22} \omega_{v}{ }^{2}\right) d u d v .
\end{aligned}
$$

This equation is in the same form as equation (5), and from this point on the argument is so nearly the same as in the nonparametric case that it need not be repeated here. The analogue of inequality (10) is seen to be

$$
\begin{aligned}
H_{11}\left(x, y, z, x_{u}, \cdots, z_{v} ; \lambda\right) H_{22}\left(x, y, z, x_{u}, \cdots, z_{v} ; \lambda\right) & \\
& -H_{12}{ }^{2}\left(x, y, z, x_{u}, \cdots, z_{v} ; \lambda\right) \geqq 0 .
\end{aligned}
$$

Columbia University.

\section{NOTE ON THE DERIVATIVE AND THE VARIATION OF A FUNCTION DEPENDING ON ALL THE VALUES OF ANOTHER FUNCTION.}

BY PROFEsSor G. C. EVANS.

(Read before the American Mathematical Society, January 2, 1915.)

1. IN a recent article,* Fréchet has given a treatment of the differential of a function depending on a curve, by making use of and evaluating Riesz's expression of a linear relation in terms of a Stieltjes integral. According to Fréchet, if $F\left[\begin{array}{c}b \\ a\end{array}\right]$ depends on all the values of $\varphi(x)$ between $a$ and $b$, then

* M. Fréchet, "Sur la notion de différentielle d'une fonction de ligne," Transactions of the American Mathematical Society, vol. 15 (1914), pp. $135-161$. 